\title{
Diagnosis of Chronic Idiopathic Inflammatory Bowel Disease Using Bayesian Networks
}

\author{
Nicandro Cruz-Ramírez ${ }^{1}$, Héctor-Gabriel Acosta-Mesa ${ }^{1}$, \\ Rocío-Erandi Barrientos-Martínez ${ }^{1}$, and Luis-Alonso Nava-Fernández ${ }^{2}$ \\ ${ }^{1}$ Facultad de Física e Inteligencia Artificial, Universidad Veracruzana, Sebastián Camacho \\ 5, Col. Centro, C.P. 91000, Xalapa, Veracruz, México \\ ${ }^{2}$ Instituto de Investigaciones en Educación, Universidad Veracruzana, Diego Leño 8 esq. \\ Morelos, Col. Centro, C.P. 91000, Xalapa, Veracruz, México \\ \{ncruz, heacosta, lunava\} @uv.mx, \\ erandi_bm@yahoo.com.mx
}

\begin{abstract}
In this paper, we evaluate the effectiveness of four Bayesian network classifiers as potential tools for the histopathological diagnosis of chronic idiopathic inflammatory bowel disease (CIIBD) using a database containing endoscopic colorectal biopsies. CIIBD is the generic term for referring to two ailments known as Crohn's disease and ulcerative colitis. The results show that the defined histological attributes, considered relevant in the medical literature for the diagnosis of CIIBD, are very good for the distinction between normal samples and CIIBD samples (Crohn's disease and ulcerative colitis combined into a single category) but less good for the explicit distinction between Crohn's disease and ulcerative colitis. The findings suggest an intrinsic impossibility of selecting a set of features for achieving good balance for both sensitivity and specificity for Crohn's disease and ulcerative colitis.
\end{abstract}

\section{Introduction}

Chronic idiopathic inflammatory bowel disease (CIIBD) is the generic term for referring to two disorders of the digestive tract known as Crohn's disease and ulcerative colitis (UC) $[5,12,15,16,19,21]$. Both of them are characterized by acute and chronic inflammation of the bowel. Crohn's disease involves the small intestine at its junction with the large intestine, or the small and the large intestines or the colon alone; the ulcerative colitis is restricted to the large intestine. Because the two diseases involve common symptoms, the distinction in the diagnosis of one or another is not an easy task. Needless to say, the correct diagnosis is imperative for a proper patient management, which, among other things, can help treat the disease properly and reduce the danger for the patient to develop cancer. Actually, there are two important distinctions in making the diagnosis: a) does the patient have CIIBD or not? b) If the patient has CIIBD, is it Crohn's disease or ulcerative colitis? In the first distinction, this implies that the patient receives/does not receive a long-term follow-up. Such a followup might include a colonoscopy every year; a procedure that unfortunately has a small associated mortality, high discomfort for the patient and high cost. However, if a patient who has CIIBD is not treated, it is possible that she/he could develop colorectal 
carcinoma. In the second distinction, this implies that the patient, who has ulcerative colitis and is not responding to the medical therapy, can have her/his colon removed. If the patient has Crohn's disease, such a surgical removal is not recommended.

Histopathology is considered the gold-standard technique for the diagnosis of CIIBD [5]. However, it is important to mention that the accurate diagnosis of CIIBD, by means of histopathology, heavily relies on the histopathologist's expertise $[5,19]$. This situation can be perceived when noticing the fact that the time it normally takes in some countries (such as United Kingdom) to a medical doctor to become an independent histopathologist is about 5 years as a minimum [5]. Such a fact gives certain indication of the very complex learning process through which medical doctors have to pass and the subjective nature of the histopathological diagnosis. In other words, a well-trained eye is needed for the correct and consistent diagnosis of CIIBD. Regarding this subjectivity, it is often the case that even expert histopathologists disagree on the interpretation of a sample; the so-called interobserver variability problem. This might suggest that there could be many factors responsible for impeding the reproducibility of the results, ranging from their expertise and the amount of stress and tiredness to the level of diligence when looking at the specimens. Due to the mentioned situations, a natural and sensible question arises: is it possible to construct computational support tools that help reduce this subjectivity? In this paper, we will explore the possibility of extracting some "objective" features, from a database, relevant for determining the presence/absence of CIIBD using a graphical-modeling approach called Bayesian networks [20]. Such an approach allows both the visual representation of the probabilistic interactions among variables of interest and the quantitative measure of the impact of those interactions. These two important properties permit to perform some inferential processes, such as prognosis and diagnosis. In order to do this, we assess the performance of four Bayesian network classifiers so as to determine their effectiveness for accurately diagnosing CIIBD using a real-world dataset.

Although some researchers have analyzed the same datasets using different techniques such as logistic regression and GCS (growing cell structure) [5], there are no studies, to the best of our knowledge, involving the analysis of CIIBD using Bayesian networks. The double nature of Bayesian-network classifiers (qualitative and quantitative) [9] can give some other insights of the phenomenon under study. For instance, an intrinsic limitation of logistic regression is the change in the classification accuracy if different orderings of the explanatory variables are considered [4]. Bayesian networks not only permit to visualize the interactions among variables but also provide local probability distributions which, despite their numerical nature, can be traced from beginning to end so that it is possible to know why and how a Bayesian network arrived at a specific conclusion. Furthermore, in contrast to logistic regression, the Bayesian network classifiers we use here are not sensitive to variable ordering.

The remainder of this paper is organized as follows. In section 2 , we present the materials and methods used for the experiments reported here. In section 3 we present the experimental methodology and the results. In section 4, we provide a discussion of these results and finally, in section 5, we give some conclusions and mention future work. 


\section{Materials and Methods}

\subsection{The Datasets}

The real-world database for this study comes from the field of histopathological diagnosis of endoscopic colorectal biopsies received at the Department of Histopathology, Royal Hallamshire Hospital in Sheffield, United Kingdom, between 1990 and 1995 inclusive. This database is partitioned in three subsets. The first one is the complete dataset (called "All IBD \& Normal"), which contains 809 cases of which 165 are normal, 473 are ulcerative colitis (UC) and 171 are Crohn's disease. The second one ("All IBD”) contains 644 cases of which 473 are UC and 171 Crohn's disease. The third one ("Active IBD") contains 370 cases of which 283 are active UC and 87 active Crohn's disease ("active" refers to the presence of active inflammation as indicated by polymorphs in the lamina propria). 23 independent variables and one dependent variable (the outcome) form part of these datasets. The diagnosis was confirmed by endoscopy, radiology and microbiological culture results. Continuous variables were

Table 1. The observed features of CIIBD

\begin{tabular}{|l|l|}
\hline Variable & Type \\
\hline 1. Age & Real number \\
\hline 2. Sex & Binary \\
\hline 3. Active inflammation & Binary \\
\hline 4. Mucosal surface & Ordinal, categorical \\
\hline 5. Crypt architecture & Ordinal, categorical \\
\hline 6. Crypt profiles & Real number \\
\hline 7. Increased lamina propria cellularity & Binary \\
\hline 8. Superficially increased lamina propria cellularity & Binary \\
\hline 9. Increased lymphoid aggregates & Binary \\
\hline 10. Patchy increased lamina propria cellularity & Binary \\
\hline 11. Transmucosally lamina propria cellularity & Binary \\
\hline 12. Cryptitis: extent & Ordinal, categorical \\
\hline 13. Cryptitis: polymorphs & Ordinal, categorical \\
\hline 14. Crypt abscesses: extent & Ordinal, categorical \\
\hline 15. Crypt abscesses: polymorph & Ordinal, categorical \\
\hline 16. Increased lamina propria polymorphs & Binary \\
\hline 17. Epithelial changes & Ordinal, categorical \\
\hline 18. Mucin depletion & Ordinal, categorical \\
\hline 19. Intraepithelial lymphocytes & Binary \\
\hline 20. Subepithelial collagen & Binary \\
\hline 21. Lamina propria granulomas & Binary \\
\hline 22. Submucosal granulomas & Binary \\
\hline 23. Basal histiocytes & Binary \\
\hline 24. Outcome & Binary \\
\hline
\end{tabular}


discretized using the CAIM algorithm [18]. Table 1 briefly explains the meaning and types of each of the variables. Most of them have to do with the dimension, architecture and counts of the cells coming from endoscopic colorectal biopsies. For more details on these variables, the reader is referred to [15].

\subsection{Bayesian Network Classifiers}

Classification refers to the task of assigning class labels to unlabelled instances. In other words, given a set of cases having values for each variable or attribute (including a special kind called class), we have to build a model (in our case a Bayesian network) that best describes the class or concept using the rest of the attributes. Once this is done, the model is used for classifying new cases that do not have a label for the class. This kind of learning is known as supervised learning [8, 13, 17]. For the sake of brevity, we do not write here the code of the 4 procedures used in the tests carried out in this work. Instead, we only describe them briefly and refer the reader to their original sources. The procedures used in these tests are: a) the Naïve Bayes classifier, b) MP-Bayes, c) Greedy and d) algorithm B [1, 2, 6, 7].

1. The Naïve Bayes classifier (NB) is one of the most effective classifiers $[7,8,13]$ and against which state of the art classifiers have to be compared. Its main appeals are its simplicity and accuracy: although its structure is always fixed (the class variable has an arc pointing to every attribute), it has been shown that this classifier has a high classification accuracy and optimal Bayes' error [7, 13]. In simple terms, the NB learns, from a training data sample, the conditional probability of each attribute given the class. Then, once a new case arrives, the NB uses Bayes' rule to compute the conditional probability of the class given the set of attributes selecting the value of the class with the highest posterior probability.

2. MP-Bayes [6] is a constraint-based algorithm that uses information measures for building a Bayesian network structure from data. Its parsimonious nature allows it to represent the joint probability distribution underlying the data with the least number of arcs.

3. Greedy [6] is a search and scoring algorithm, which uses greedy-hill climbing for the search part and the Bayesian Information Criterion (BIC) metric for the scoring part $[10,11,14]$. For the experiments reported here, procedure Greedy takes as input an empty graph and a database. Other alternatives for the initialization space are a complete graph, a random graph or a graph suggested by a constraint-based procedure. In every search step, it looks for a graph that minimizes the BIC score. Such an algorithm is a modification of that proposed by Friedman et al. [8]. In brief, procedure Greedy applies, whenever possible, 3 different operators: the addition of an arc (in either direction), the deletion of an arc and the reversal of an arc. In every step, the BIC score is calculated and procedure Greedy keeps the structure with the best (minimum) score. It finishes searching when no structure improves the BIC score of the previous network.

4. Power Constructor is a software package which contains two different algorithms (algorithms A and B) to build BN structures from data [1]. For the tests carried out here, we used algorithm B because it does not need a node ordering to work. Algorithm $\mathrm{B}$ uses mutual information and conditional mutual information tests to decide when to connect/disconnect a pair of nodes. 
We have selected different types of Bayesian network classifiers: the Naïve Bayesian classifier, two constraint-based classifiers (b, and d) and one search and scoring classifier c). We have chosen them to check the behavior and performance of different statistical measures, heuristics and metrics in classification tasks.

\section{Experimental Methodology and Results}

In this section, we describe the experimental methodology and present the results for 3 different experiments. In section 4, we discuss such results. Because of the lack of space, the Bayesian network structures resultant from running these databases are not presented.

Experiment 1. We used the "All IBD and Normal" database split into 2 subsets: the training and the test sets. The size of the training set is $2 / 3$ of the data (540 cases) and the remaining $1 / 3$ of the data forms the test set. All the algorithms described in the previous section use these training data to learn a classification model. Then, they use such a classification model and the test set to compute two tests, commonly used in the medical domain, for comparing the performance of these four classifiers: sensitivity (the ability to correctly identify those patients who actually have the disease) and specificity (the ability to correctly identify those patients who do not have the disease). The central goal of Experiment 1 is to assess the discriminant power of the independent variables to tell normal samples from CIIBD samples (Crohn's disease and UC are combined into a single category). The results of experiment 1 are shown in table 2, which shows the sensitivity and specificity for Normal vs. CIIBD samples. For these tests, their respective $95 \%$ confidence intervals (CI) are shown in parentheses.

Table 2. IBD vs. Normal's sensitivity and specificity with their respective $95 \%$ confidence intervals in the "All IBD vs. Normal" dataset

\begin{tabular}{|l|c|c|c|c|}
\hline & Naïve-Bayes & MP-Bayes & Greedy & Algorithm B \\
\hline Sensitivity & $96 \%(92-100)$ & 0 & 0 & 0 \\
\hline Specificity & $69 \%(63-75)$ & $100 \%(100)$ & $100 \%(100)$ & $100 \%(100)$ \\
\hline
\end{tabular}

Experiment 2. We used the "All IBD" database split into 2 subsets: the training and the test sets. The size of the training set is $2 / 3$ of the data (430 cases) and the remaining $1 / 3$ of the data forms the test set. All the algorithms described in the previous section use these training data to learn a classification model. Then, they use such a classification model and the test set to compute sensitivity and specificity tests. The main objective of Experiment 2 is to assess the discriminant power of the independent variables to tell Crohn's disease samples from UC samples. The results of experiment 2 are shown in tables 3 and 4, which show the sensitivity and specificity for Crohn's disease and UC respectively. For these tests, their respective 95\% confidence intervals (CI) are shown in parentheses. 
Table 3. Crohn's disease's sensitivity and specificity with their respective $95 \%$ confidence intervals in the "All IBD" dataset

\begin{tabular}{|c|c|c|c|c|}
\hline & Naïve-Bayes & MP-Bayes & Greedy & Algorithm B \\
\hline Sensitivity & $65 \%(53-78)$ & $16 \%(7-26)$ & $20 \%(9-31)$ & $27 \%(16-39)$ \\
\hline Specificity & $68 \%(61-75)$ & $93 \%(89-97)$ & $96 \%(92-99)$ & $94 \%(90-97)$ \\
\hline
\end{tabular}

Table 4. UC's sensitivity and specificity with their respective $95 \%$ confidence intervals in the "All IBD" dataset

\begin{tabular}{|c|c|c|c|c|}
\hline & Naïve-Bayes & MP-Bayes & Greedy & Algorithm B \\
\hline Sensitivity & $68 \%(61-75)$ & $93 \%(89-97)$ & $96 \%(92-99)$ & $94 \%(90-97)$ \\
\hline Specificity & $65 \%(53-78)$ & $16 \%(7-26)$ & $20 \%(9-31)$ & $27 \%(16-39)$ \\
\hline
\end{tabular}

Experiment 3. We used the "Active IBD" database split into 2 subsets: the training and the test sets. The size of the training set is $2 / 3$ of the data ( 247 cases) and the remaining $1 / 3$ of the data forms the test set. All the algorithms described in the previous section use these training data to learn a classification model. Then, they use such a classification model and the test set to compute sensitivity and specificity tests. The main objective of Experiment 3 is to assess the discriminant power of the independent variables to tell active Crohn's disease samples from active UC samples. The results of experiment 3 are shown in tables 5 and 6 , which show the sensitivity and specificity for active Crohn's disease and active UC respectively. For these tests, their respective $95 \%$ confidence intervals (CI) are shown in parentheses.

Table 5. Crohn's disease's sensitivity and specificity with their respective $95 \%$ confidence intervals in the "Active IBD" dataset

\begin{tabular}{|l|c|c|c|c|}
\hline & Naïve-Bayes & MP-Bayes & Greedy & Algorithm B \\
\hline Sensitivity & $57 \%(39-75)$ & $14 \%(1-27)$ & $14 \%(1-27)$ & $14 \%(1-27)$ \\
\hline Specificity & $72 \%(63-81)$ & $99 \%(97-100)$ & $99 \%(97-100)$ & $99 \%(97-100)$ \\
\hline
\end{tabular}

Table 6. UC's sensitivity and specificity with their respective $95 \%$ confidence intervals in the "Active IBD" dataset

\begin{tabular}{|c|c|c|c|c|}
\hline & Naïve-Bayes & MP-Bayes & Greedy & Algorithm B \\
\hline Sensitivity & $72 \%(63-81)$ & $99 \%(97-100)$ & $99 \%(97-100)$ & $99 \%(97-100)$ \\
\hline Specificity & $57 \%(39-75)$ & $14 \%(1-27)$ & $14 \%(1-27)$ & $14 \%(1-27)$ \\
\hline
\end{tabular}

\section{Discussion of the Results}

According to the medical literature, all 22 defined histological observations mentioned in table 1 are considered relevant for the histopathological diagnosis of CIIBD [4]. Age is also considered relevant as it provides useful information for making the final diagnosis. All variables are random variables and take on values as described in 
table 1 . In the experiments we call such variables a1, a2, and so on. The 4 procedures presented here select the relevant attributes according to their own metrics, as described in section 2.2. Now, let us describe our findings for the described experiments.

Experiment 1. The central goal of Experiment 1 is to assess the discriminant power of the independent variables to tell normal samples from CIIBD samples. Table 2 shows a high sensitivity value for the NB classifier (96\%) but $0 \%$ sensitivity for the remaining classifiers. This significant difference strongly suggests that it is important to consider all the attributes in order to achieve high sensitivity values. If only few attributes are considered relevant for explaining the outcome (a7 for MP-Bayes; a2, a3 and a4 for Greedy; and a5 and a16 for procedure B), $0 \%$ sensitivity is achieved. On the other hand, a regular specificity value is achieved by NB $(69 \%)$ but a perfect specificity value $(100 \%)$ is reached by the remaining classifiers. The attributes selected by MP-Bayes, Greedy and procedure B (a7; a2, a3 and a4; and a5 and a16 respectively) as the most discriminant attributes for the absence of CIIBD seem to work excellently. This supports the findings in Cross and Harrison [5]: histopathological examination of endoscopic colorectal biopsies is very good for the distinction between Normal samples and CIIBD samples. It is important to mention that the human expert achieves $82 \%$ for sensitivity and $99 \%$ for specificity [5] for this same database. This important finding suggests that the expert is taking into account more information than that portrayed by the variables in table 1 for making the final decision; otherwise, the NB classifier would perform as well as he does.

Experiment 2. The main objective of Experiment 2 is to assess the discriminant power of the independent variables to tell Crohn's disease samples from UC samples (see tables 3 and 4). Both tables 3 and 4 show a regular performance of the NB classifier in terms of sensitivity and specificity (65\%-68\% and 68\%-65\% respectively). These results seem to indicate that the consideration of all attributes for deciding whether a sample belongs to one or the other disease is insufficient. The human expert reaches a sensitivity value of $44 \%$ and a specificity value of $99 \%$ for the same experiment of table 3 and a sensitivity value of $58 \%$ and a specificity value of $99 \%$ for the same experiment of table 4 [3]. These results also support the findings in Cross and Harrison [5]: histopathological examination of endoscopic colorectal biopsies is less good for the distinction between Crohn's disease and UC than that between Normal samples and CIIBD samples. Furthermore, in the case of specificity, it also seems that the expert is taking into account more information than that portrayed by the attributes in table 1 for making the final decision. The remaining classifiers select the following attributes as the most discriminant ones: a1 and a2 for the case of MPBayes; a1, a2, a4 and a21 for Greedy; and a1, a5 and a21 for procedure B. This selection is very bad for detecting the presence of Crohn's disease (sensitivity values of $16 \%, 20 \%$ and $27 \%$ ) but excellent for detecting its absence (specificity values of $93 \%$, $96 \%$ and $94 \%$; see table 3). This same selection shows the opposite result for detecting the presence of UC (sensitivity values of $93 \%, 96 \%$ and $94 \%$ ) and for detecting its absence (specificity values of $16 \%, 20 \%$ and $27 \%$; see table 4 ). Such mixed results reveal the difficulty in differentiating samples belonging to one or the other disease.

Experiment 3. The main objective of Experiment 3 is to assess the discriminant power of the independent variables to tell active Crohn's disease samples from active 
UC samples (see tables 5 and 6). Both tables show a regular performance of the NB classifier in terms of sensitivity and specificity (57\%-72\% and $72 \%-57 \%$ respectively). Once again, these results seem to indicate that the consideration of all attributes for deciding whether a sample belongs to one or the other active disease is insufficient. The human expert reaches a sensitivity value of $30 \%$ and a specificity value of $93 \%$ for the same experiment of table 5 and a sensitivity value of $61 \%$ and a specificity value of $76 \%$ for the same experiment of table 6 [5]. These results also support the findings in Cross and Harrison [5]: histopathological examination of endoscopic colorectal biopsies is less good for the distinction between active Crohn's disease and active UC than that between Normal samples and CIIBD samples. Furthermore, in the case of specificity, it also seems that the expert is taking into account more information than that portrayed by the attributes in table 1 for making the final decision. The remaining classifiers select the following attributes as the most discriminant ones: a21 for the case of MP-Bayes; a10 and a21 for Greedy; and a10 and a21 for procedure B. This selection is very bad for detecting the presence of active Crohn's disease (sensitivity values of $14 \%$ for all the classifiers) but excellent for detecting its absence (specificity values of $99 \%$ for all the classifiers; see table 5). This same selection shows the opposite result for detecting the presence of active UC (sensitivity values of $99 \%$ for all the classifiers) and for detecting its absence (specificity values of $14 \%$ for all the classifiers; see table 6). Such mixed results reveal the difficulty in differentiating samples belonging to one or the other active disease.

\section{Conclusions and Future Work}

In this paper, we have explored the possibility of extracting some "objective" features, from a database, relevant for determining the presence/absence of CIIBD using the Bayesian network framework. The results show that, in sum, the attributes selected by procedures MP-Bayes, Greedy and B allow us to accurately detect the absence of CIIBD. On the other hand, the results also show that it is much more difficult to clearly differentiate samples belonging to Crohn's disease or to ulcerative colitis. Thus, such results suggest an intrinsic impossibility of selecting a set of features for achieving good balance for both sensitivity and specificity for Crohn's disease and $\mathrm{UC}$, given that the only information we can use is that portrayed by the attributes. In other words, these results seem to indicate that the attributes in table 1 are not enough for making the final diagnosis and that the human experts are taking into account more information for arriving to their conclusions.

In the paper by Cross and Harrison [5], they run the same tests described in tables 2 and 6 using logistic regression and a technique known as GCS (growing cell structure); this last technique is described in [22]. The sensitivity and specificity results for logistic regression running the same tests as in table 2 are $78 \% \%$ and $89 \%$ respectively. The results for these same tests for GCS are $76 \%$ and $86 \%$. The sensitivity and specificity results for logistic regression running the same tests as in table 6 are $84 \%$ and $62 \%$ respectively. The results for these same tests for GCS are $66 \%$ and $66 \%$. It is important to mention the root of such differences: while in a Bayesian network different value combinations of the same set of variables determine whether it is a normal sample or a CIIBD sample (or whether it is Crohn's disease or UC), in the logistic 
regression and in GCS different sets of variables determine the presence or absence of the disease being considered. As can be inferred, different combinations of such variables for both techniques, as well as the order of the explanatory variables for logistic regression, are a key ingredient for obtaining good/bad results. In the case of procedures MP-Bayes, Greedy and B, there is no prior knowledge that privileges specific variables in the analysis; i.e., not a single variable is treated with a special distinction. Moreover, the very nature of these three procedures is to follow Occam's razor: they keep the least number of arcs among variables. The Bayesian network structures not only show the interactions between the attributes and the class but also the interactions among the attributes themselves. Such features may be helpful for both describing the phenomenon under study and classifying new samples with unknown class label. In contrast, logistic regression and GCS only allow the classification of new samples but do not provide a description of the phenomenon under investigation.

It is important to keep in mind that the process or processes that histopathologists follow to make their final diagnoses have not been yet fully understood and can only be partially explained in terms of pattern recognition with occasional use of heuristic logic. Hence, the information provided there is subjective rather than objective. To ameliorate this problem, alternative data collection methods such as image analysis techniques could be used so that objective measures from sample raw digitalized images can be extracted. In sum, our contribution has mainly to do with the discovery of an intrinsic impossibility of manually selecting a set of features for achieving a good balance for sensitivity and specificity in Crohn's disease and UC. We plan to investigate the possibility of building a pre-processing vision module capable of extracting "objective" features from raw images and, again, check the performance of the Bayesian network classifiers using the dataset produced by this module.

\section{Acknowledgements}

We are very grateful to Dr. Simon S. Cross, Clinical Senior Lecturer at the Academic Unit of Pathology of the University of Sheffield, United Kingdom, who provided the databases used in this study.

\section{References}

1. Cheng, J., Bell, D.A., Liu, W. Learning Belief Networks from Data: An Information Theory Based Approach. in Sixth ACM International Conference on Information and Knowledge Management. 1997: ACM.

2. Cheng, J. and R. Greiner. Comparing Bayesian Network Classifiers. in Fifteenth Conference on Uncertainty in Artificial Intelligence. 1999.

3. Cross, S.S., Dataset of Observed Features on Endoscopic Colorectal Biopsies from Normal Subjects and Patients with Chronic Inflammatory Bowel Disease (Crohn's disease and U1cerative Colitis). 1999, Department of Pathology, University of Sheffield Medical School. p. $1-15$.

4. Cross, S.S., et al., Which Decision Support Technologies Are Appropriate for the Cytodiagnosis of Breast Cancer?, in Artificial Intelligence Techniques in Breast Cancer Diagnosis and Prognosis, A. Jain, et al., Editors. 2000, World Scientific. p. 265-295. 
5. Cross, S.S. and H. R.F., Discriminant histological features in the diagnosis of chronic idiopathic inflammatory bowel disease: analysis of a large dataset by a novel data visualisation technique. Journal of Clinical Pathology, 2002(55): p. 51-57.

6. Cruz-Ramirez Nicandro, N.-F.L., Acosta-Mesa Hector Gabriel, Barrientos-Martinez Erandi, Rojas-Marcial Juan Efrain, A Parsimonious Constraint-based Algorithm to Induce Bayesian Network Structures from Data, in IEEE Proceedings of the Mexican International Conference on Computer Science ENC 2005, IEEE, Editor. 2005, IEEE: Puebla. p. 306-313.

7. Duda, R.O., Hart, Peter E., Stork, David G., Pattern Classification. 2001: John Wiley \& Sons, INC.

8. Friedman, N., D. Geiger, and M. Goldszmidt, Bayesian Network Classifiers. Machine Learning, 1997. 29: p. 131-163.

9. Friedman, N. and M. Goldszmidt, Learning Bayesian Networks from Data. 1998, University of California, Berkeley and Stanford Research Institute. p. 117.

10. Grunwald, P., Introducing MDL, in Advances in Minimum Description Length: Theory and Applications, P. Grunwald, I.J. Myung, and M.A. Pitt, Editors. 2005, The MIT Press.

11. Grunwald, P., Tutorial on MDL, in Advances in Minimum Description Length: Theory and Applications, P. Grunwald, I.J. Myung, and M.A. Pitt, Editors. 2005, The MIT Press.

12. Guindi, M. and R.H. Riddell, Indeterminate colitis. Journal of Clinical Pathology, 2004(57): p. 1233-1244.

13. Han, J. and M. Kamber, Data Mining. Concepts and Techniques. First ed. Data Management Systems, ed. J. Gray. 2001: Morgan Kaufmann. 550.

14. Heckerman, D., A Tutorial on Learning with Bayesian Networks, in Learning in Graphical Models, M.I. Jordan, Editor. 1998, MIT Press. p. 301-354.

15. Jenkins, D., et al., Guidelines for the initial biopsy diagnosis of suspected chronic idiopathic inflammatory bowel disease. The British Society of Gastroenterology Initiative. Journal of Clinical Pathology, 1997(50): p. 93-105.

16. Jenkins, D., et al., What is colitis? Statistical approach to distinguishing clinically important inflammatory change in rectal biopsy specimens. Journal of Clinical Pathology, 1988(41): p. 72-79.

17. Kohavi, R. A Study of Cross-Validation and Bootstrap for Accuracy Estimation and Model Selection. in 14th International Joint Conference on Artificial Intelligence IJCAI'95. 1995a. Montreal, Canada: Morgan Kaufmann.

18. Kurgan, L.A., Cios, Krzysztof J., CAIM Discretization Algorithm. IEEE Transactions on Knowledge and Data Engineering, 2004. 16(2): p. 145-153.

19. Lessells, A.M., et al., Observer variability in the histopathological reporting of abnormal rectal biopsy specimens. Journal of Clinical Pathology, 1994(47): p. 48-52.

20. Pearl, J., Probabilistic Reasoning in Intelligent Systems: Networks of Plausible Inference. First ed. Representation and Reasoning, ed. R.J.B.A.T.B. Laboratories). 1988, San Mateo, California: Morgan Kaufmann. 552.

21. Sheperd, N.A., Pathological mimics of chronic inflammatory bowel disease. Journal of Clinical Pathology, 1991(44): p. 726-733.

22. Walker, A.J., S.S. Cross, and R.F. Harrison, Visualisation of biomedical datasets by use of growing cell structure networks: a novel diagnostic classification technique. The Lancet, 1999. 354: p. 1518-1521. 